\title{
DEPOSIÇÃO DE SERAPILHEIRA E NUTRIENTES POR ESPÉCIES NATIVAS EM UMA FLORESTA ESTACIONAL DECIDUAL EM ITAARA, RS, BRASIL
}

\author{
Hamilton Luiz Munari Vogel ${ }^{1}$, Mauro Valdir Schumacher ${ }^{2}$, Peter Trüby ${ }^{3}$ \\ Eng. Florestal, Dr. UNIPAMPA, São Gabriel, RS, Brasil - hamiltonvogel@ unipampa.edu.br \\ ${ }^{2}$ Eng. Florestal, Dr., Depto. de Ciências Florestais, UFSM, Santa Maria, Brasil - mvschumacher@gmail.com \\ ${ }^{3}$ Eng. Florestal, Dr., Albert-Ludwigs-Universität Freiburg, Freiburg, Alemanha - peter.trueby@ bodenkunde.uni-freiburg.de
}

Recebido para publicação: 04/05/2011 - Aceito para publicação: 11/08/2011

\begin{abstract}
Resumo
O presente trabalho foi realizado em uma Floresta Estacional Decidual no município de Itaara (RS), Brasil, e teve como principal objetivo avaliar a produção de folhas e a transferência ao solo de nutrientes de sete espécies arbóreas nativas. Para a coleta das folhas, foram demarcadas seis parcelas de $25 \mathrm{~m} \mathrm{x} 17$ $\mathrm{m}$ cada, alocadas no interior da floresta. Em cada parcela foram distribuídos cinco coletores, totalizando assim 30 coletores. As coletas foram realizadas mensalmente, durante um período de 2 anos. No laboratório, o material foi separado, levando em consideração apenas as espécies selecionadas: Ocotea puberula (A. Rich.) Nees, Ocotea pulchella Mart., Nectandra megapotamica (Spreng.) Mez, Matayba elaeagnoides Radlk., Cupania vernalis Cambess., Parapiptadenia rigida (Benth.) Brenan e Schinus molle L. Nesse material, foram determinados os teores de N, P, K, Ca, Mg, S, Fe, Mn e Zn. Os resultados evidenciaram que houve variação da quantidade de deposição de folhas e nutrientes sobre o solo da floresta entre as espécies nativas analisadas. A espécie que mais transferiu nutrientes (principalmente N, P, K, Ca e S) foi a Parapiptadenia rigida.

Palavras-chave: Floresta nativa; folhas; ciclagem de nutrientes.
\end{abstract}

\begin{abstract}
Leaves deposition and nutrients of tree species in a native forest in southern Brazil. The present study was conducted in a fragment of a seasonal deciduous forest in Itaara (State of Rio Grande do Sul, Brazil). This research has as its main aim to evaluate the monthly leaves production and nutrients transfer to the soil of seven native species. Six plots $(25 \mathrm{~m} \mathrm{x} 17 \mathrm{~m})$ were delimited to collect the deposited leaves, with five round collectors in each plot, in a total of 30 collectors. The deposited leaves had been collected monthly during a period of 24 months. In such material it was determined the content of nutrients as $\mathrm{N}, \mathrm{P}, \mathrm{K}, \mathrm{Ca}, \mathrm{Mg}, \mathrm{S}, \mathrm{Fe}, \mathrm{Mn}$ and $\mathrm{Zn}$. In laboratory, the leaves were identified into seven native species: Ocotea puberula (A. Rich.) Nees, Ocotea pulchella Mart., Nectandra megapotamica (Spreng.) Mez, Matayba elaeagnoides Radlk., Cupania vernalis Cambess., Parapiptadenia rigida (Benth.) Brenan and Schinus molle L. The results revealed a variation in deposition in relation to the amount of leaves as well as in forest soil among the native species analyzed. The tree species which transferred more litter and nutrients (mainly N, P, K, Ca and S) was Parapiptadenia rigida.

Keywords: Native forest; leaves; nutrients cycling.
\end{abstract}

\section{INTRODUÇÃO}

O estudo da ciclagem de nutrientes (ciclo biogeoquímico) desempenha um papel importante no conhecimento das condições e dinâmica dos processos internos dos ecossistemas naturais, que auxiliam no entendimento das mudanças provocadas pela exploração florestal no ambiente (FEGER; RASPE, 1998), sendo o mesmo imprescindível nos estudos de nutrição, transferências e exportação de nutrientes em florestas.

No Brasil, os estudos envolvendo os ecossistemas naturais, com ênfase na ciclagem de nutrientes em florestas nativas, surgem da necessidade de se ampliar e aprofundar os conhecimentos sobre os aspectos nutricionais em plantações florestais com espécies nativas, tendo em vista sua utilização nas atividades aplicadas à implantação de maciços puros ou mistos destinados à recuperação de áreas 
degradadas ou mesmo o cultivo de essências nativas para produção de madeiras de lei (POGGIANI; SCHUMACHER, 2000).

Binkley (1986) comenta que a entrada de nutrientes pela atmosfera e intemperismo de rochas é mais importante a longo prazo no desenvolvimento de solos e ecossistemas, mas a ciclagem interna de nutrientes é a principal forma de liberação de nutrientes para as plantas. As raízes das árvores retiram os minerais das camadas mais profundas do solo (especialmente as árvores decíduas, que possuem um extenso e profundo sistema radicular), armazenando temporariamente em sua biomassa, e finalmente devolvendo os nutrientes pela deposição de material vegetal sobre o solo (LARCHER, 2000). O tipo de vegetação e as condições ambientais são os fatores que mais influem na quantidade e qualidade do material que cai no solo (MOREIRA; SIQUEIRA, 2002).

A quantidade de material orgânico depositado ao longo de um ano está relacionada principalmente com as condições climáticas, sendo menor nas regiões frias e maior nas regiões equatoriais quentes e úmidas (POGGIANI; SCHUMACHER, 2000). Por exemplo, florestas situadas em regiões árticas ou alpinas produzem anualmente cerca de uma tonelada de serapilheira por hectare; florestas temperadas frias, 3,5 toneladas; florestas temperadas quentes, 5,5 toneladas; florestas equatoriais, cerca de 11 toneladas (BRAY; GORHAM, 1964).

Essa quantidade de material vegetal que cai do dossel formando a serapilheira em Florestas Estacionais no sul do Brasil atinge várias toneladas por hectare, conforme vários trabalhos já realizados pelos autores Cunha (1997), Barichello et al. (2000), König et al. (2002), Vogel et al. (2003), Caldeira (2003), Brun (2004) e Pereira et al. (2008). Reissmann e Wisniewski (2000) ressaltam que a absorção de nutrientes diretamente da serapilheira representa um fluxo importante para atender a demanda nutricional das árvores. Dessa maneira, a serapilheira em um ecossistema florestal é responsável pela retenção de grandes quantidades de nutrientes, sendo uma fonte importante de devolução de nutrientes para o solo (ANDRAE; KRAPFENBAUER, 1983; REIS; BARROS, 1990; BRUN et al., 1999; ALBERTI et al. 2000; SCHUMACHER et al., 2002; TOLEDO et al., 2002; VOGEL et al., 2003; CALDEIRA, 2003; BRUN, 2004; VITAL et al., 2004; CALDEIRA et al., 2006), constituindo uma via importante do ciclo biogeoquímico, especialmente em solos altamente intemperizados, onde a biomassa vegetal é o principal reservatório de nutrientes (REIS; BARROS, 1990).

Apesar dos vários estudos já realizados com serapilheira em florestas nativas, são escassos os trabalhos que visam o conhecimento de quais as espécies arbóreas que mais contribuem para a devolução de folhas e, consequentemente, de nutrientes para o solo da floresta, sendo esta a principal hipótese que norteou o presente trabalho.

Assim, o presente estudo teve como principais objetivos a avaliação da deposição mensal de folhedo de diferentes espécies arbóreas nativas e a quantificação dos nutrientes transferidos ao solo por essas espécies, em uma Floresta Estacional Decidual na região de Itaara (RS), no sul do Brasil.

\section{MATERIAL E MÉTODOS}

O trabalho foi realizado em uma fazenda da Fundação da Brigada Militar, no município de Itaara, estado do Rio Grande do Sul, Brasil. O município de Itaara está localizado na Serra de São Martinho, na região fisiográfica do Planalto Médio. Verifica-se, na figura 1, a localização do município de Itaara no estado do Rio Grande do Sul e da área experimental com as coordenadas médias de $29^{\circ} 30^{\prime} 12,2^{\prime \prime} \mathrm{S}$ e $53^{\circ} 46^{\prime} 06^{\prime \prime} \mathrm{O}$. A altitude média do local é de $400 \mathrm{~m}$.

A região em que o município de Itaara está inserido apresenta clima Cfa (classificação climática de Köppen). De acordo com a plataforma de coleta de dados do Instituto Nacional de Pesquisas Espaciais (INPE) de São Martinho da Serra/RS (estação mais próxima da área experimental), no período compreendido entre 2003 e 2006 a temperatura média foi de $19,6{ }^{\circ} \mathrm{C}$ e a precipitação anual de $1.520,5 \mathrm{~mm}$. Ainda conforme Moreno (1961), na região, a temperatura média do mês mais quente é superior a $22{ }^{\circ} \mathrm{C}$ e a do mês mais frio entre 18 e $-3{ }^{\circ} \mathrm{C}$, com a ocorrência de geadas no inverno.

O solo da região é caracterizado como Neossolo Litólico Eutrófico típico (STRECK et al., 2008). Essa classe de solos é descrita pela Empresa Brasileira de Pesquisa Agropecuária (EMBRAPA) (1999) como "solos com horizonte A ou O hístico com menos de $40 \mathrm{~cm}$ de espessura, assente diretamente sobre a rocha ou sobre um horizonte $\mathrm{C}$ ou $\mathrm{Cr}$ ou sobre material com $90 \%$ ou mais de sua massa, constituída por fragmentos de rocha com diâmetro maior que $2 \mathrm{~mm}$ (cascalhos, calhaus e matacões)". Ainda conforme a EMBRAPA (1999), esses solos têm alta saturação de bases (V $\geq 50 \%)$ em todos os horizontes, o que também foi constatado pela análise de solo do local, sendo o mesmo considerado de 
fertilidade média a alta. Também é importante ressaltar que, no local do estudo, o solo é bastante raso, ficando em torno de $35 \mathrm{~cm}$ de profundidade.

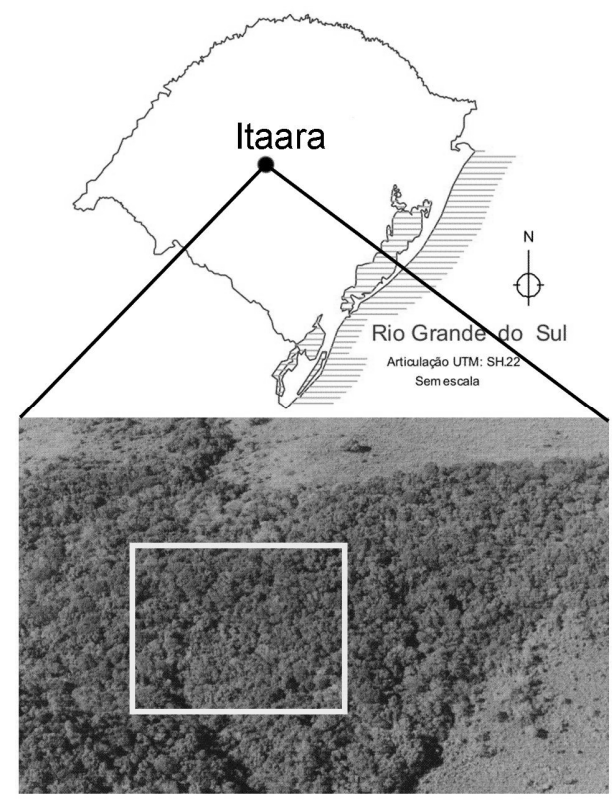

Figura 1. Localização do município de Itaara e da área experimental no estado do Rio Grande do Sul, Brasil.

Figure 1. Location of the city of Itaara and the experimental area in the State of the Rio Grande do Sul, Brazil.

Quanto à composição florística do local, de acordo com o inventário realizado por Greff $e t$ al. (2006), foram encontradas 52 espécies, divididas em 24 famílias, destacando-se as famílias Leguminosae, Euphorbiaceae e Sapindaceae, devido aos seus representantes apresentarem os maiores valores no Índice de Valor de Importância (IVI) (estimadores da importância ecológica de um táxon - espécie, família etc. dentro de uma comunidade florestal). A família com maior número de representantes foi a Myrtaceae, porém todos eles com baixos valores nos índices relativos. As espécies de maior destaque foram a Parapiptadenia rigida e a Sebastiana commersoniana, devido a seus altos valores no IVI, que totalizam 48,73\%; a Parapiptadenia rigida apresentou o maior valor de dominância (área basal de determinada espécie por unidade de área) e a Sebastiana commersoniana a maior densidade e frequência (GREFF et al., 2006). Quanto à idade e estágio de desenvolvimento, conforme as informações da Fundação da Brigada Militar, responsável pelo local, estima-se que a floresta tenha em torno de 70 anos de idade e esteja em estágio secundário.

Para a coleta das folhas depositadas, foram demarcadas seis parcelas de $25 \mathrm{~m} \times 17 \mathrm{~m}$ cada, alocadas no interior da floresta. Em cada parcela, foram distribuídos cinco coletores, totalizando assim trinta coletores. Estes são constituídos por uma moldura de ferro de forma circular, com $50 \mathrm{~cm}$ de diâmetro, fixados no local, com uma altura de um metro partindo do solo. Em cada coletor, prendeu-se uma tela de sombrite com malha de $2 \mathrm{~mm}$. As coletas das folhas foram realizadas mensalmente, durante um período de 24 meses (de dezembro de 2002 a novembro de 2004). Todo o material depositado mensalmente nos coletores foi acondicionado, identificado e levado até o Laboratório de Ecologia Florestal do Departamento de Ciências Florestais da Universidade Federal de Santa Maria (UFSM). Na sequência, nas folhas da serapilheira depositada, foram separadas as folhas de sete espécies arbóreas nativas. As espécies identificadas foram: Ocotea puberula (A. Rich.) Nees, Ocotea pulchella Mart., Nectandra megapotamica (Spreng.) Mez, Matayba elaeagnoides Radlk., Cupania vernalis Cambess., Parapiptadenia rigida (Benth.) Brenan e Schinus molle L. A identificação dessas espécies foi realizada em função da frequência e quantidade de suas folhas na serapilheira depositada nos coletores. Após a separação, o material foi colocado em estufa a $75^{\circ} \mathrm{C}$ até atingir massa constante. Na sequência, o material foi moído em moinho do tipo Willey e foi determinada a massa, com balança digital de $0,01 \mathrm{~g}$ de precisão, obtendo-se assim a massa seca.

As análises químicas foram realizadas no Laboratório do Institut für Bodenkunde und Waldernährungslehre (Instituto de Ciência do Solo e Nutrição de Florestas) da Universidade Albert- 
Ludwig, de Freiburg, na Alemanha. Nas análises químicas, foram determinados os teores totais de N, P, $\mathrm{K}, \mathrm{Ca}, \mathrm{Mg}, \mathrm{S}, \mathrm{Fe}, \mathrm{Mn}$ e Zn. A metodologia utilizada foi a digestão em ácido nítrico e perclórico, em sistema fechado com micro-ondas, sendo o nitrogênio medido por cromatografia gasosa em analisador CNS da Carlo Erba NA 1500, e os teores totais de P, K, Ca, Mg, S, Fe, Mn e Zn foram determinados em aparelho ICP (Inductiv Coppled Plasma). O estoque de nutrientes nas folhas das espécies foi obtido com base na biomassa seca estimada, multiplicada pelo teor de nutrientes do respectivo componente.

\section{RESULTADOS E DISCUSSÃO}

Na figura 2, é possível verificar a variação da produção de folhas de cada uma das sete espécies arbóreas nativas analisadas, ao longo dos 24 meses de coleta. Nota-se, nessa figura, que a deposição de folhas variou conforme a espécie.

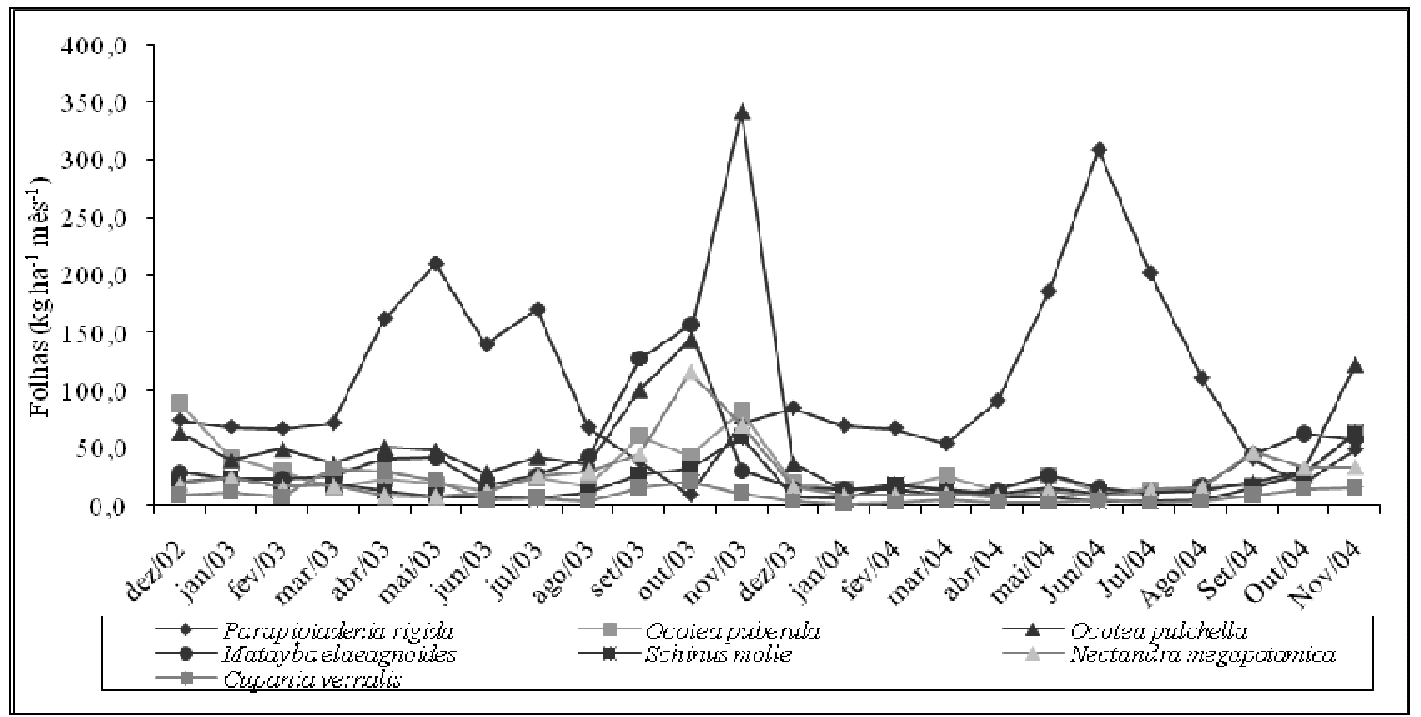

Figura 2. Produção mensal de folhas pelas espécies estudadas na Floresta Estacional Decidual de Itaara, RS, Brasil.

Figure 2. Monthly leaves production of the studied species in Seasonal Decidual Forest in Itaara, RS, Brazil.

A espécie que mais produziu folhas foi a Parapiptadenia rigida (Figura 2). Essa espécie teve as máximas deposições no período de abril a agosto/2003 e de maio a agosto/2004, com pico no mês de junho/2004, o que indica um padrão de sazonalidade ao longo do ano (Figura 2).

As demais espécies mantiveram um comportamento sazonal mais ou menos semelhante, com pequenas variações, com as maiores deposições nos meses de setembro a novembro, com exceção da Ocotea pulchella, que teve uma grande queda de folhas no mês de novembro de 2003 (Figura 2).

Na tabela 1, pode-se visualizar o total anual de folhedo depositado em $\mathrm{kg}$ por hectare e a percentagem relativa da produção de folhas pelas espécies analisadas. Constata-se nessa tabela que $23,1 \%$ das folhas foram produzidas por Parapiptadenia rigida, seguida de Ocotea pulchella, com 12,3\% (na floresta estudada, a Parapiptadenia rigida é uma das espécies que ocorre com maior frequência), estabelecendo-se a seguinte ordem de participação na devolução de folhas: Parapiptadenia rigida > Ocotea pulchella > Matayba elaeagnoides $>$ Ocotea puberula $>$ Nectandra megapotamica $>$ Schinus molle $>$ Cupania vernalis.

A variação na produção de folhas pelas espécies ao longo do ano pode estar associada com a fenologia de cada espécie e as condições climáticas da região (Tabela 1). O ano de 2003 foi mais quente e chuvoso do que o ano de 2004, conforme dados climáticos do INPE de São Martinho da Serra (estação climática próxima da área do estudo). No ano de 2003 , a temperatura média anual foi de $18,1{ }^{\circ} \mathrm{C}$ e a precipitação total de $1.684,3 \mathrm{~mm}$, ao passo que no ano de 2004 a temperatura média anual foi de $16,9{ }^{\circ} \mathrm{C}$ e a precipitação total de $921,3 \mathrm{~mm}$. Portanto, em 2003 houve maior produção de folhas com temperatura e precipitação mais elevadas do que em 2004, o que pode sugerir a maior queda de folhas em $2003 \mathrm{em}$ função dessas características climáticas. 
Tabela 1. Média anual e participação relativa das espécies na produção de folhas na Floresta Estacional Decidual em Itaara, RS, Brasil, ao longo de 2003 e 2004.

Table 1. Annual average relative share of species, and leaves production in Seasonal Decidual Forest in Itaara, RS, Brazil, during 2003 and 2004.

\begin{tabular}{lcccccccc}
\hline & \multicolumn{7}{c}{ Espécies arbóreas } \\
\hline \multirow{3}{*}{$\begin{array}{l}\text { Média anual } \\
\text { \% }\end{array}$} & P. r. & O. pr. & O. pch. & M. e. & S. . & N. m. & C. v. & FNI \\
\cline { 2 - 9 } & 1213,5 & 354,9 & 645,0 & 434,3 & 209,6 & 302,5 & 121,5 & 1969,5 \\
CV $(\%)^{*}$ & 23,1 & 6,8 & 12,3 & 8,3 & 4,0 & 5,8 & 2,3 & 37,5 \\
\hline P. & 70,9 & 74,8 & 131,8 & 99,2 & 88,9 & 97,6 & 85,2 & 35,3 \\
\hline
\end{tabular}

P. r.: Parapiptadenia rígida; O. pr.: Ocotea puberula; O. pch.: Ocotea pulchella; M. e.: Matayba elaeagnoides; S. m.: Schinus molle; N. m.: Nectandra megapotamica; C. v.: Cupania vernalis; FNI: folhas não identificadas; ${ }^{*} \mathrm{CV}$ : coeficiente de variação.

Na sazonalidade da produção de serapilheira na floresta, também se deve levar em conta a fenologia particular de cada espécie e suas características genéticas específicas.

Cunha (1997), na área de um capoeirão com 19 anos, na Floresta Estacional Decidual em São João do Polesine (RS), verificou que as espécies que mais produziam folhas eram Ocotea puberula, Cupania vernalis e Parapiptadenia rigida, indicando que a fenologia particular de cada espécie e a sua densidade na floresta desempenham um papel decisivo no funcionamento do ecossistema, no que diz respeito à devolução de folhas. Dependendo da composição florística das espécies na floresta, ela acarretará maior ou menor devolução de folhas e, consequentemente, uma maior ou menor devolução de nutrientes para a floresta, apontando a importância de se conhecer as espécies arbóreas que compõem a floresta.

Quanto aos nutrientes, na tabela 2 estão os teores totais de N, P, K, Ca, Mg, S, Mn, Fe e Zn nas folhas das espécies arbóreas nativas depositadas no solo da floresta estudada. Pode-se constatar, de modo geral, que os teores nas folhas variam entre as espécies analisadas.

Tabela 2. Teores médios de nutrientes nas folhas produzidas pelas diferentes espécies arbóreas nos dois anos de coleta (dez/02-nov/04) na Floresta Estacional Decidual em Itaara, RS, Brasil.

Table 2. Nutrient content in the leaves by the different tree species produced during the two years of sampling (Dec/02-Nov/04) in Seasonal Decidual Forest in Itaara, RS, Brazil.

\begin{tabular}{|c|c|c|c|c|c|c|c|c|c|}
\hline \multirow{2}{*}{ Espécie } & $\mathbf{N}$ & $\mathbf{P}$ & $\mathbf{K}$ & $\mathbf{C a}$ & Mg & $\mathbf{S}$ & Mn & Fe & Zn \\
\hline & \multicolumn{6}{|c|}{ 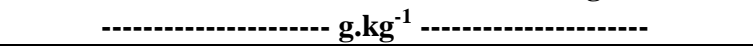 } & \multicolumn{3}{|c|}{------ mg.kg-1 -----.- } \\
\hline O. pr. & 26,7 & 1,3 & 4,8 & 11,2 & 1,5 & 1,9 & 98,8 & 100,8 & 52,0 \\
\hline O. pc. & 19,3 & 1,0 & 3,4 & 11,4 & 1,5 & 1,4 & 166,8 & 93,3 & 39,3 \\
\hline N. m. & 20,1 & 1,0 & 4,4 & 14,7 & 2,4 & 1,4 & 59,4 & 75,7 & 52,7 \\
\hline M. e. & 20,8 & 1,2 & 7,6 & 12,2 & 6,3 & 2,0 & 332,8 & 88,9 & 40,5 \\
\hline C. v. & 22,0 & 1,1 & 6,0 & 14,1 & 4,1 & 2,0 & 353,0 & 97,3 & 61,8 \\
\hline P. r. & 29,0 & 1,5 & 3,5 & 18,6 & 2,3 & 1,8 & 85,2 & 126,3 & 44,2 \\
\hline S. m. & 16,2 & 1,1 & 5,8 & 12,1 & 2,5 & 1,4 & 70,5 & 83,9 & 55,8 \\
\hline FNI & 22,1 & 1,3 & 6,2 & 19,5 & 3,2 & 1,7 & 178,1 & 147,7 & 47,2 \\
\hline $\mathrm{CV}(\%)$ & 18,5 & 13,5 & 28,0 & 22,7 & 53,8 & 15,3 & 69,10 & 23,40 & 15,85 \\
\hline
\end{tabular}

O. pr.: Ocotea puberula; O. pch.: Ocotea pulchella; N. m.: Nectandra megapotamica; M. e.: Matayba elaeagnoides; C. v.: Cupania vernalis; P. r.: Parapiptadenia rígida; S. m.: Schinus molle; FNI: folhas não identificadas.

Em relação aos teores de nutrientes, pode-se verificar na tabela 2 que os maiores teores de $\mathrm{N}, \mathrm{P}$, $\mathrm{Ca}$ e $\mathrm{Fe}$ foram encontrados na espécie Parapiptadenia rigida. Os maiores teores de $\mathrm{K}$ e $\mathrm{Mg}$ estão na espécie Matayba elaeagnoides. Já os maiores teores de S, Mn e $\mathrm{Zn}$ foram encontrados na espécie Cupania vernalis.

$\mathrm{Na}$ literatura, são poucos os trabalhos com dados de teores de nutrientes nas folhas de espécies nativas. Cunha (1997) obteve os seguintes teores para as espécies arbóreas em uma Floresta Estacional Decidual em São João do Polesine (RS): Parapiptadenia rigida: $23,3 \mathrm{~g} \cdot \mathrm{kg}^{-1}$ de N, 1,9 g.kg ${ }^{-1}$ de P, 4,8 g.kg ${ }^{-1}$ de K, 19,1 g. $\mathrm{kg}^{-1}$ de Ca e 2,0 g.kg ${ }^{-1}$ de Mg; Ocotea puberula: 18,7 g.kg ${ }^{-1}$ de N, 2,2 g.kg-1 de P, 4,8 g.kg-1 de K, 12,4 g.kg $\mathrm{kg}^{-1}$ de $\mathrm{Ca}$ e 1,4 g.kg ${ }^{-1}$ de Mg; Cupania vernalis: 15,3 g.kg ${ }^{-1}$ de N, 1,9 g.kg ${ }^{-1}$ de $\mathrm{P}$, 7,4 g. $\mathrm{kg}^{-1}$ de K, 16,9 g.kg ${ }^{-1}$ de Ca e 3,9 g.kg ${ }^{-1}$ de Mg. Já Hiremath et al. (2002) determinaram, em três espécies tropicais, os teores de $\mathrm{P}$ e $\mathrm{N}$ nas folhas: Hyeronima alchorneoides Fr. All., com 18,5 g. $\mathrm{kg}^{-1}$ de $\mathrm{N}$ e 1,7 g. kg ${ }^{-1}$ de P, Cedrela odorata L., com 23,4 g.kg ${ }^{-1}$ de N e 2,7 g. $\mathrm{kg}^{-1}$ de P e Cordia alliodora (Ruiz \& Pav.) Oken., com 25,9 g.kg ${ }^{-1}$ de $\mathrm{N}$ e 2,7 g.kg ${ }^{-1}$ de P. 
Quanto aos micronutrientes Mn, Fe e Zn, em nível de comparação, Caldeira et al. (2006), em uma floresta Ombrófila Mista Montana em General Carneiro/PR, encontraram os seguintes teores nas folhas de Cupania vernalis: $927,67 \mathrm{mg} \cdot \mathrm{kg}^{-1}$ de $\mathrm{Mn}, 29,10 \mathrm{mg} \cdot \mathrm{kg}^{-1}$ de $\mathrm{Zn} \mathrm{e} 151,67 \mathrm{mg} \cdot \mathrm{kg}^{-1}$ de Fe; Matayba elaeagnoides: $763,0 \mathrm{mg} \cdot \mathrm{kg}^{-1}$ de $\mathrm{Mn}, 22,85 \mathrm{mg} \cdot \mathrm{kg}^{-1}$ de $\mathrm{Zn} \mathrm{e} 130,5 \mathrm{mg} \cdot \mathrm{kg}^{-1}$ de Fe; Ocotea puberula: $355,33 \mathrm{mg} \cdot \mathrm{kg}^{-1}$ de Mn, 30,56 mg. $\mathrm{kg}^{-1}$ de Zn e $166,73 \mathrm{mg} \cdot \mathrm{kg}^{-1}$ de Fe; Ocotea pulchella: $496,5 \mathrm{mg} \cdot \mathrm{kg}^{-1} \mathrm{de}$ Mn, 13,46 mg. $\mathrm{kg}^{-1}$ de Zn e 137,55 mg.kg-1 de Fe.

$\mathrm{Na}$ tabela 3 é apresentada a quantidade de nutrientes (kg/ha/ano) de N, P, K, Ca, Mg, S, Mn, Fe e $\mathrm{Zn}$ transferidos pela queda das folhas das diferentes espécies para o solo da floresta.

Tabela 3. Quantidade média anual de nutrientes depositados pelas diferentes espécies arbóreas por ha/ano, durante os 24 meses da pesquisa realizada, na Floresta Estacional Decidual em Itaara, RS, Brasil.

Table 3. Mean quantity of annual nutrient deposition by different tree species per ha/year during the 24months of study (Dec 02-Nov 04) in Seasonal Decidual Forest in Itaara, RS, Brazil.

\begin{tabular}{|c|c|c|c|c|c|c|c|c|c|}
\hline \multirow{3}{*}{ Espécie } & $\mathbf{N}$ & $\mathbf{P}$ & K & Ca & Mg & $\mathbf{S}$ & Mn & $\mathbf{F e}$ & Zn \\
\hline & \multirow{2}{*}{\multicolumn{9}{|c|}{ Ano 2003 (dez/02-nov/03) }} \\
\hline & & & & & & & & \\
\hline O. pr. & 13,1 & 0,6 & 1,9 & 5,7 & 0,6 & 0,8 & 0,04 & 0,04 & 0,02 \\
\hline O. pc. & 18,3 & 1,0 & 2,6 & 11,8 & 1,3 & 1,1 & 0,15 & 0,34 & 0,03 \\
\hline N. m. & 7,9 & 0,4 & 1,6 & 5,9 & 0,8 & 0,5 & 0,01 & 0,03 & 0,02 \\
\hline M. e. & 11,8 & 0,7 & 4,9 & 6,6 & 3,8 & 1,0 & 0,21 & 0,04 & 0,02 \\
\hline C. v. & 4,0 & 0,2 & 1,1 & 2,5 & 0,8 & 0,3 & 0,07 & 0,02 & 0,01 \\
\hline P.r. & 34,5 & 1,9 & 3,4 & 19,2 & 2,3 & 2,0 & 0,07 & 0,12 & 0,03 \\
\hline S. m. & 3,9 & 0,3 & 1,4 & 2,8 & 0,6 & 0,3 & 0,01 & 0,02 & 0,00 \\
\hline FNI & 55,2 & 3,2 & 13,8 & 39,3 & 7,1 & 3,6 & 0,44 & 0,29 & 0,08 \\
\hline Total & 148,7 & 8,3 & 30,7 & 93,8 & 17,3 & 9,6 & 1,00 & 0,90 & 0,21 \\
\hline \multicolumn{10}{|c|}{ Ano 2004 (dez/03-nov/04) } \\
\hline O. pr. & 6,8 & 0,3 & 1,2 & 2,9 & 0,4 & 0,6 & 0,03 & 0,03 & 0,02 \\
\hline O. pc. & 5,5 & 0,2 & 1,1 & 3,8 & 0,5 & 0,5 & 0,06 & 0,03 & 0,01 \\
\hline N. m. & 3,9 & 0,2 & 1,0 & 3,3 & 0,7 & 0,3 & 0,03 & 0,02 & 0,02 \\
\hline M. e. & 5,4 & 0,3 & 2,1 & 4,1 & 1,7 & 0,6 & 0,09 & 0,03 & 0,01 \\
\hline C. v. & 1,4 & 0,1 & 0,5 & 1,1 & 0,3 & 0,1 & 0,03 & 0,01 & 0,00 \\
\hline P.r. & 34,0 & 1,5 & 4,2 & 27,9 & 3,1 & 2,3 & 0,12 & 0,19 & 0,06 \\
\hline S. m. & 2,4 & 0,1 & 1,1 & 2,3 & 0,5 & 0,3 & 0,02 & 0,02 & 0,01 \\
\hline FNI & 36,9 & 1,7 & 10,5 & 42,0 & 6,5 & 3,2 & 0,39 & 0,29 & 0,10 \\
\hline Total & 96,3 & 4,4 & 21,7 & 87,4 & 13,7 & 7,9 & 0,77 & 0,62 & 0,23 \\
\hline
\end{tabular}

O. pr.: Ocotea puberula; O. pch.: Ocotea pulchella; N. m.: Nectandra megapotamica; M. e.: Matayba elaeagnoides; C. v.: Cupania vernalis; P. r.: Parapiptadenia rígida; S. m.: Schinus molle; FNI: folhas não identificadas.

Observa-se, na tabela 3, que no primeiro ano do estudo a Parapiptadenia rigida foi a espécie que mais transferiu os elementos $\mathrm{N}, \mathrm{P}, \mathrm{Ca}$ e S para o solo da floresta. No segundo ano, essa espécie manteve tal tendência, devolvendo as maiores quantidades de $\mathrm{N}, \mathrm{P}, \mathrm{K}, \mathrm{Ca}, \mathrm{Mg}$ e S.

As maiores quantidades de $\mathrm{K}, \mathrm{Mg}$ e $\mathrm{Mn}$ foram transferidas ao solo pelas folhas da espécie Matayba elaeagnoides durante o primeiro ano. As maiores quantidades de $\mathrm{Fe}$ foram transferidas pelas folhas da Ocotea pulchella.

Observa-se na tabela 3 que, de modo geral, grandes quantidades de nutrientes são transferidos para o solo da floresta via deposição de folhas, sobretudo o $\mathrm{N}$ e o Ca. No segundo ano, a transferência de nutrientes foi menor, devido à menor queda de folhas nesse período. Ao longo dos 24 meses, o total de $\mathrm{N}$ devolvido pelas folhas chegou a $148,6 \mathrm{~kg} \cdot \mathrm{ha}^{-1}$ no primeiro ano e $96,3 \mathrm{~kg} \cdot \mathrm{ha}^{-1}$ no segundo ano. Cunha (1997) quantificou para uma floresta secundária com mais de 30 anos com condições semelhantes de fenologia, que a transferência de $\mathrm{N}$ pelas folhas das árvores foi de $110 \mathrm{~kg} \cdot \mathrm{ha}^{-1}$ por ano.

Assim, pode-se indicar a seguinte ordem de contribuição por espécie na devolução de nutrientes pelas folhas: Parapiptadenia rigida $>$ Ocotea pulchella $>$ Matayba elaeagnoides $>$ Ocotea puberula $>$ Nectandra megapotamica $>$ Schinus molle $>$ Cupania vernalis.

Os resultados obtidos neste estudo indicam que existe variação entre as espécies nativas analisadas, em relação à transferência ao solo de nutrientes pelas folhas. Em parte, essas diferenças 
podem ser atribuídas às características fisiológicas e genéticas específicas de cada espécie, como a absorção, distribuição e utilização dos nutrientes pela planta.

Dessa maneira, estes resultados podem fornecer subsídios para o manejo de espécies nativas, como a introdução adequada de espécies em sítios florestais, visando a recuperação da fertilidade do solo. Nesse sentido, a espécie Parapiptadenia rigida destaca-se entre as demais, pois, além de ser uma espécie leguminosa que fixa nitrogênio, retém em suas folhas teores elevados de nutrientes, podendo devolver grandes quantidades de $\mathrm{N}, \mathrm{P}, \mathrm{K}, \mathrm{Ca}$ e $\mathrm{S}$ para o solo da floresta.

\section{CONCLUSÕES}

- Por meio deste estudo, foi possível determinar a quantidade dos diferentes nutrientes transferidos para o solo da floresta pelas principais árvores da floresta estudada.

- A Parapiptadenia rigida destaca-se das demais pelos teores elevados de nutrientes, principalmente $\mathrm{N}, \mathrm{P}, \mathrm{K}, \mathrm{Ca}$ e S, transferidos para o solo da floresta.

- Estes resultados auxiliam no manejo sustentado de florestas, fornecendo subsídios, como a escolha de espécies que melhor se adaptam a determinados sítios, composição de maciços florestais puros ou mistos e a recuperação de áreas degradadas nutricionalmente ou erodidas.

\section{AGRADECIMENTOS}

O primeiro autor agradece à Capes, pela bolsa de estudos de doutorado no Brasil, e o DAAD, pela bolsa de estudos na Alemanha.

\section{REFERÊNCIAS}

ALBERTI, L. F.; SCHUMACHER, M. V.; BARICHELLO, L. R.; VOGEL, H. L. M.; D'AVILA, M.; CANTARELI, E. B. Análise de solo e serapilheira em floresta Estacional Decidual, no município de Santa Maria, RS. In: CONGRESSO FLORESTAL ESTADUAL, 8., 2000, Nova Prata. Anais do... Nova Prata: UFSM, 2000. 1 CD-ROM.

ANDRAE, F.; KRAPFENBAUER, A. Inventário de um reflorestamento de Araucária de 17 anos em Passo Fundo-RS: Inventário da biomassa e nutrientes. In: ANDRAE, F.; KRAPFENBAUER, A. Pesquisas Austríaco-Brasileiras 1973-1982. Wien, 1983. p. 16 - 55.

BARICHELLO, L. R.; SCHUMACHER, M. V.; VOGEL, H. L. M.; DALLAGO, J. S. Quantificação dos nutrientes no solo e serapilheira de diferentes estágios sucessionais em um sistema de agricultura migratória. In: REUNIÃO SUL-BRASILEIRA DE CIÊNCIA DO SOLO, 3., 2000, Pelotas. Anais do... Pelotas: Embrapa Clima Temperado, 2000. 1 CD-ROM.

BINKLEY, D. Forest nutrition management. New York: J. Wiley \& Sons, 1986. 290 p.

BRAY, J. R.; GORHAM, E. Litter production in forests of the world. Advances in Ecological Research, London, v. 2, p. $101-157,1964$.

BRUN, E. J. Biomassa e nutrientes na floresta Estacional Decidual, em Santa Tereza, RS. Santa Maria: UFSM, 2004. 136 f. Dissertação (Mestrado em Engenharia Florestal) - Universidade Federal de Santa Maria, Santa Maria, 2004.

BRUN, E. J.; SCHUMACHER, M. V.; VACCARO, S. Produção de serapilheira e devolução de nutrientes em três fases sucessionais de uma Floresta Estacional Decidual no município de Santa Tereza (RS). In: SIMPÓSIO DE FERTILIZAÇÃO E NUTRIÇÃO FLORESTAL, 1999, Piracicaba. Anais do... Piracicaba: ESALQ, 1999. 1 CD-ROM.

CALDEIRA, M. V. W. Determinação de biomassa e nutrientes em uma floresta Ombrófila Mista Montana em General Carneiro, Paraná. Curitiba: UFPR, 2003. 176 f. Tese - (Doutorado em Ciências Florestais) - Universidade Federal do Paraná, Curitiba, 2003.

CALDEIRA, M. V. W.; WATZLAWICK, L. F.; SOARES, R. V.; VALÉRIO, A. F. Teores de micronutrientes em espécies arbóreas da floresta Ombrófila Mista Montana, General Carneiro/PR. Ambiência, Guarapuava, v. 2, n. 1, p. 29 - 50, 2006. 
CUNHA, G. C. Aspectos da ciclagem de nutrientes em diferentes fases sucessionais de uma Floresta Estacional do Rio Grande do Sul. Piracicaba: Escola Superior de Agricultura "Luiz de Queiroz", 1997. 86 f. Dissertação (Mestrado em Engenharia Florestal) - Escola Superior de Agricultura "Luiz de Queiroz", Piracicaba, 1997.

EMBRAPA. Centro Nacional de Pesquisa de Solos (Rio de Janeiro, RJ). Sistema brasileiro de classificação de solos. Rio de Janeiro, 1999. 412 p.

FEGER, K. H.; RASPE, S. Ökosystemforschung im chwarzwald: Auswirkungen von atmogenen einträgen und Restabilisierungsmassnahmen auf den Wasser- und Stoffhaushalt von Fichtenwäldern. In: RASPE, FEGER UND ZÖTTL (Hrsg). Verbundprojekt ARINUS: Landsberg: Umweltforschung in Baden-Württenberg. Freiburg, 1998. p. 1 - 18.

GREFF, L. T. B.; LONGHI, S. J.; BRUN, F. G. K.; BRUN, E. J.; FREITAG, Â. S.; BERGER, G. Estrutura fitossociológica de um fragmento de Floresta Estacional Decidual no município de Itaara, RS. In: SIMPÓSIO DE PÓS-GRADUAÇÃO EM CIÊNCIAS FLORESTAIS, 4., 2006. Piracicaba-SP. Anais do... Piracicaba: ESALQ, 2006. 1 CD-ROM.

HIREMATH, A. J.; EWEL, J. J.; COLE, T. G. Nutrient use efficiency in three fast-growing tropical trees. Forest Science, Bethesda, v. 48, n. 4, p. 662 - 672, 2002.

KÖNIG, F. G.; SCHUMACHER, M. V.; BRUN, E. J.; SELING, I. Avaliação da sazonalidade da produção de serapilheira numa floresta Estacional Decidual no município de Santa Maria, RS. Revista Árvore, Viçosa-MG, v. 26, n. 4, p. 429 - 435, 2002.

LARCHER, W. Ecofisiologia vegetal. São Carlos: RiMa, 2000. 531 p.

MOREIRA, F. M. S.; SIQUEIRA, J. O. Microbiologia e bioquímica do solo. Lavras: Ed. UFLA, 2002. 626 p.

MORENO, J. A. Clima do Rio Grande do Sul. Porto Alegre: Secretaria da Agricultura, 1961. 73 p.

POGGIANI, F.; SCHUMACHER, M. V. Ciclagem de nutrientes em Florestas Nativas. In: GONÇALVES, J. L. M.; BENEDETTI, V. (eds.). Nutrição e fertilização florestal. Piracicaba: IPEF, 2000. p. 287 - 308.

PEREIRA, M. G.; MENEZES, L. F. T.; SCHULTZ, N. Aporte e decomposição da serapilheira na floresta Atlântica, ilha da Marambaia, Mangaratiba, RJ. Ciência Florestal, Santa Maria, RS, v. 18, n. 4, p. 443 454, 2008.

REIS, M. G. F.; BARROS, N. F. Ciclagem de nutrientes em plantios de eucalipto. In: BARROS, N. F.; NOVAIS, R. F. (eds.). Relação solo-eucalipto. Viçosa: Ed. Folha de Viçosa, 1990. p. 265 - 302.

REISSMANN, C. B.; WISNIEWSKI, C. Aspectos nutricionais de plantios de Pinus. GONÇALVES, J. L.; BENEDETTI, V. (eds.). Nutrição e fertilização florestal. Piracicaba: IPEF, 2000. p. 135 - 166.

SCHUMACHER, M. V.; CALDEIRA, M. V. W.; SPATHELF, P.; CAPRA, A. Quantifizierung der Biomasse und dês Nährstoffgehalts bei der Restdurchforstung eines Araukarienbestandes in Quedas do Iguaçu (Paraná, Brasilien). Forstarchiv, Stuttgart, v 73, p. 187 - 194, 2002.

STRECK, E. V.; KÄMPF, N.; DALMOLIN, R. S. D.; KLAMT, E.; NASCIMENTO, P. C; SCHNEIDER, P. GIASSON, E; PINTO, L. F. S. Solos do Rio Grande do Sul. Porto Alegre: EMATER/RS; 2 ed. UFRGS, 2008. 222 p.

TOLEDO, L. O.; PEREIRA, M. G.; MENEZES, C. E. G. Produção de serapilheira e transferência de nutrientes em florestas secundárias localizadas na região de Pinheiral, RJ. Ciência Florestal, Santa Maria, v. 12, n. 2, p. 9 - 16, 2002.

VITAL, A. R. T.; GUERRINI, I. A.; FRANKEN, W. K.; FONSECA, R. C. B. Produção de serapilheira e ciclagem de nutrientes de uma Floresta Estacional Semidecidual em Zona Ripária. Revista Árvore, Viçosa-MG, v. 28, n. 6, p. 793 - 800, 2004.

VOGEL, H. L. M.; SCHUMACHER, M. V.; LOPES, V. G. Biomassa e nutrientes na serapilheira de uma floresta Estacional Decidual. In: CONGRESSO FLORESTAL ESTADUAL DO RIO GRANDE DO SUL, 9., 2003, Nova Prata. Anais do... Nova Prata: UFSM, 2003. 1 CD-ROM. 